\title{
Different Model for End-Stage Liver Disease Score Block Distributions May Have a Variable Ability for Outcome Prediction
}

\author{
Teh-Ia Huo, ${ }^{1,2,4}$ Han-Chieh Lin, ${ }^{1,2}$ Jaw-Ching Wu, ${ }^{1,3}$ Fa-Yauh Lee, ${ }^{1,2}$ Ming-Chih Hou, ${ }^{1,2}$ \\ Pui-Ching Lee, ${ }^{2}$ Full-Young Chang, ${ }^{1,2}$ and Shou-Dong Lee ${ }^{1,2}$
}

\begin{abstract}
Background. The model for end-stage liver disease (MELD) scoring system has become the prevailing criteria for organ allocation in liver transplantation. However, it is not clear if the predictive accuracy of MELD is equally homogeneous in different distribution of MELD score blocks.

Methods. We investigated 472 cirrhotic patients (mean MELD, 14.3 \pm 5.5 ), and compared the predictive accuracy of MELD and the corresponding Child-Turcotte-Pugh (CTP) scores in patients with low $(<16)$, intermediate $(10-20)$ and high $(>14)$ MELD score range by using c-statistic for area under the receiver operating characteristic curve (AUC) at different time frames.

Results. The MELD scores well correlated with CTP scores at baseline $(\rho=0.492, P<0.001)$. Overall, MELD was significantly better than the CTP system to predict the risk of mortality. However, in stratified analysis there were no significant differences between MELD and CTP for the c-statistic in patients with low and intermediate range MELD scores at 3-, 6-, 9-, and 12-month ( $\mathrm{p}$ values all $>0$ 1). Among patients with high MELD scores, MELD was consistently more accurate than the CTP system in predicting the mortality at 3 - (AUC, 0.715 vs. $0.543, P=0.020), 6-(0.705$ vs. $0.536, P=0.003), 9$ - ( 0.737 vs. $0.507, P<0.001)$ and 12 -month ( 0.716 vs. $0.526, P<0.001)$, respectively.

Conclusions. MELD has a better performance only in a subset of patients with higher MELD scores. The outcome in patients with lower range MELD scores cannot be reliably predicted solely with their MELD scores, and alternative prognostic markers should be used in conjunction to enhance the predictive accuracy.
\end{abstract}

Keywords: Child-Turcotte-Pugh, Liver cirrhosis, Liver transplantation, MELD.

(Transplantation 2005;80: 1414-1418)

$\mathrm{P}$ atients with decompensated liver cirrhosis often have a limited survival, and liver transplantation is the only definite treatment modality to effectively prolong their lifespan. However, patients on the waiting list of transplantation far outnumber the potential cadaveric or living liver donors. As a result, the number of patients dying while on the waiting list is progressively increasing in recent years (1).

The donor livers are allocated to the recipients according to the severity of underlying liver disease. The MELD (model for end-stage liver disease) score has been shown to more accurately predict the survival than the Child-TurcottePugh (CTP) score in a recent multi-center study in the United States (2), and the liver allocation system has changed from a status-based algorithm to one using a continuous MELD severity scale to prioritize adult patients on the waiting list (3, 4). Its accuracy for outcome prediction in patients with de-

\footnotetext{
${ }^{1}$ National Yang-Ming University School of Medicine, Taipei Veterans General Hospital, Taipei, Taiwan.

${ }^{2}$ Department of Medicine, Taipei Veterans General Hospital, Taipei, Taiwan.

${ }^{3}$ Department of Medical Research and Education, Taipei Veterans General Hospital, Taipei, Taiwan.

${ }^{4}$ Address correspondence to: Teh-Ia Huo, M.D., Division of Gastroenterology, Department of Medicine, Taipei Veterans General Hospital, Taipei, Taiwan.

E-mail: tihuo@vghtpe.gov.tw

This study was supported by a grant from the Taipei Veterans General Hospital, Taipei, Taiwan.

Received 23 March 2005. Revision requested 20 April 2005.

Accepted 29 June 2005.

Copyright $\odot 2005$ by Lippincott Williams \& Wilkins

ISSN 0041-1337/05/8010-1414

DOI: $10.1097 / 01 . t p .0000181164 .19658 .7 \mathrm{a}$
}

compensated cirrhosis has also been validated in Europe (5, $6)$.

The development of the MELD system, however, may not fairly serve all candidates for liver transplantation. The current MELD system may have potential limitations in prioritizing patients with metabolic liver diseases and other conditions $(7,8)$. In addition, patients with a lower MELD score and ascites could have a worse outcome than those with a higher MELD score without significant complications (9). Controversially, a few studies have raised a concern that the CTP system was in fact not inferior to the MELD system for outcome prediction $(10,11)$. Although the risk of mortality in cirrhotic patients is expected to increase as the MELD score increases, it is not clear if the ability of the MELD to predict the risk of mortality is equally homogeneous in different MELD score distributions for a given patient population. We have conducted this study to investigate the performance of the MELD in different score block distributions at different time frames in patients with advanced cirrhosis. Our results show that the outcome of patients with higher MELD scores can be better predicted with the MELD score compared to those with lower MELD scores.

\section{PATIENTS AND METHODS}

\section{Patients}

From January 1999 to February 2004, patients who had liver cirrhosis and visited our hospital were prospectively evaluated and their medial profiles were retrospectively analyzed in this study. The following criteria were used to select eligible patients: (1) an initial CTP score of 7 or more (equivalent to class B or C) to fulfill the minimal listing criteria for 
liver transplantation (12), (2) no coexisting hepatocellular carcinoma or human immunodeficiency virus infection at presentation, (3) known initial MELD score and survival status at 1-year of follow-up. According to these criteria, 482 patients were selected from our database. Among them, 10 patients who underwent liver transplantation within one year of their first visit were excluded from analysis. The natural history and outcome were assessed for the remaining $472 \mathrm{pa}-$ tients who formed the basis of this study. None of these patients had received specific anti-viral treatment (interferon, lamivudine, or adefovir) during the study period. This study complies with the standards of Declaration of Helsinki and current ethical guidelines.

The underlying etiology of cirrhosis was attributed to hepatitis B virus infection if patients were seropositive for hepatitis B surface antigen (HBsAg; RIA kits, Abbott Laboratories, North Chicago, IL) and attributed to hepatitis C virus (HCV) infection if patients were seropositive for antibody against $\mathrm{HCV}$ by a second-generation enzyme immunoassay (Abbott Laboratories) on at least two occasions. The diagnosis of liver cirrhosis was based on the characteristic findings including physical stigmata of cirrhosis, decreased serum albumin and increased serum globulin levels, CT or ultrasonography findings of uneven liver surface, coarsened echogenicity of liver parenchyma, enlarged spleen and/or detection of ascites, and detection of esophageal varices by endoscopy.

\section{MELD and CTP Scores}

The MELD equation used to calculate the severity score was as follows: $9.57 \times \log _{\mathrm{e}}($ creatinine $\mathrm{mg} / \mathrm{dL})+3.78 \times \log _{\mathrm{e}}$ (bilirubin $\mathrm{mg} / \mathrm{dL})+11.2 \times \log _{\mathrm{e}}(\mathrm{INR})+6.43$ (constant for liver disease etiology) (2). Minimal values are set to 1.0 for calculation purposes. The maximal serum creatinine level considered within the MELD score equation is $4.0 \mathrm{mg} / \mathrm{dL}$. The CTP score is calculated on the basis of serum bilirubin and albumin levels, the prothrombin time, and the presence and severity of ascites and encephalopathy.

\section{Statistical Methods}

Chi-squared test or Fisher's exact test (two-tailed) was used for categorical data, and Mann-Whitney ranked sum test was used for continuous data. Spearman's correlation analysis was used to estimate the correlation and significance between the MELD score and the corresponding CTP score. To investigate the impact of MELD score distribution on survival prediction, patients were partially-overlapped categorized into three groups according to their baseline MELD scores: 1) those with MELD scores $<16$ (low MELD score group); 2) those with MELD scores $>10$ and $<20$ (intermediate MELD score group); and 3) those with MELD scores $>14$ (high MELD score group). To assess the ability of MELD score and the corresponding CTP score in outcome prediction, our analysis was performed by measuring the concordance (c-statistic) equivalent to the area under the receiver operating characteristic (ROC) curve (13). Comparison of the area under ROC curves was done using the method of Hanley and McNeil (14). The outcome was assessed as 3-, 6-, 9-, and 12-month mortality. All statistical analyses were conducted using SPSS for Windows version 12 (SPSS Inc., Chicago, IL) and MedCalc for Windows version 4.2 (MedCalc Software, Mariakerke, Belgium). For all tests, a $P$ value less than 0.05 was considered statistically significant.

\section{RESULTS}

\section{Patients Demographics and Mortality}

The baseline demographics were shown in Table 1. Patients were predominantly male and had chronic hepatitis $\mathrm{B}$ virus infection. The mean MELD and CTP score was $14.3 \pm 5.5$ and $8.6 \pm 1.3$ respectively. The mortality rates at 3,6 , 9 , and 12 months in different groups of patients were given in

TABLE 1. Patient demographics

\begin{tabular}{lc}
\multicolumn{1}{c}{ Characteristics } & Data \\
\hline Number of patients & 472 \\
Age, years (mean SD) & $65 \pm 12$ \\
Male/female (\%) & $78 / 22$ \\
Etiology of cirrhosis (\%) & $73 / 27$ \\
HBsAg (+)/ HBsAg (-) & $14.3 \pm 5.5$ \\
MELD score & $12.7(6.8-38.3)$ \\
Mean \pm SD & $78 / 22$ \\
Median (range) & $8.6 \pm 1.3(7-14)$ \\
Child-Turcotte-Pugh class & $12.1 \pm 4.0(11.4,6.8-15.9)$ \\
B/C (\%) & 326 \\
Score, mean \pm SD (range) & $8.0 \pm 0.8$ \\
Groups of MELD score distribution & \\
Low (<16) score, median \pm SD (range) & $14.1 \pm 5.1(13.3,10.1-19.9)$ \\
$\quad$ No. of patients & 311 \\
Corresponding Child-Turcotte-Pugh score (mean \pm SD) & $8.7 \pm 0.7$ \\
Intermediate (10-20) score, median \pm SD (range) & 198 \\
$\quad$ No. of patients & $17.1-38.3)$ \\
Corresponding Child-Turcotte-Pugh score & $9.8 \pm 1.3$ \\
High (>14) score, median \pm SD (range) & $17.6 \pm 5.4(17.8,14$ \\
$\quad$ No. of patients &
\end{tabular}


Table 2. There was a significant trend showing the cumulative mortality rate tended to increase and reached the highest in patients with high $(>14)$ MELD scores $(P$ values all $<0.05)$; the 6 - and 12-month mortality rate in this group was up to $22.7 \%$ and $46 \%$, respectively.

\section{Correlation of MELD and Corresponding CTP Score in Different Groups}

The correlation between MELD and CTP score in all and subgroups of patients according to the MELD score were estimated and shown in Figure 1. There was a significant correlation between the MELD score and the corresponding CTP score in all patients $(\rho=0.492, P<0.001)$. The correlation was considered weak yet still significant in the subgroup of patients with low MELD score $(\rho=0.291, P<0.001)$, intermediate MELD score $(\rho=0.257, P<0.001)$ and high MELD score $(\rho=0.299, P<0.001)$.

\section{Comparison of MELD and CTP Score in Predicting Mortality in Different Score Groups and Time Frames}

Among all patients in four different time frames, MELD had a significantly better predictive accuracy of mortality than the CTP system by comparing the area under ROC curves (AUC). The AUC was $0.785,0.714,0.689$ and 0.681 for the MELD system, and was $0.635,0.607,0.594$ and 0.592 for the CTP system at 3-, 6-, 9-, and 12-month, respectively (p values all $<0.05$ in all comparisons; Figure 2). Patients were further stratified into low, intermediate and high MELD score groups to compare the respective predictive accuracy between MELD and CTP at different time periods. As shown in Figure 3, there were no significant differences of the AUC between these two models among patients with low and intermediate MELD score groups ( $\mathrm{p}$ values all $>0.1$ ). However, MELD was consistently more accurate than the CTP system in predicting the risk of mortality among patients with high MELD scores at 3 months (AUC, 0.715 vs. $0.543, P=0.020$ ), 6 months $(0.705$ vs. $0.536, P=0.003), 9$ months $(0.737$ vs. $0.507, P<0.001)$, and 12 months ( 0.716 vs. $0.526, P<0.001)$, respectively (Fig. 3 ).

\section{DISCUSSION}

The MELD scoring system has become the prevailing criteria for donor liver allocation $(3,15,16)$. It has the advantage of minimal variability and wide-range continuous scale to assess underlying disease severity compared to the traditional CTP scoring system. In addition, MELD has been shown to correlate with residual liver function and predict mortality across a broad spectrum of liver disease (17). The

TABLE 2. Mortality rate according to the distribution of MELD scores at different time frames

\begin{tabular}{lccc}
\multicolumn{1}{c}{ Mortality } & $\begin{array}{c}\text { Low }(<\mathbf{1 6}) \\
\text { score }\end{array}$ & $\begin{array}{c}\text { Intermediate } \\
(\mathbf{1 0 - 2 0}) \text { score }\end{array}$ & $\begin{array}{c}\text { High }(>\mathbf{1 4}) \\
\text { score }\end{array}$ \\
\hline $\mathrm{n}$ & 326 & 311 & 198 \\
3-month (\%) & $9(2.8)$ & $11(3.5)$ & $25(12.6)$ \\
6-month (\%) & $25(7.7)$ & $35(11.3)$ & $45(22.7)$ \\
9-month (\%) & $48(14.7)$ & $56(18)$ & $63(31.8)$ \\
12-month (\%) & $78(23.9)$ & $90(28.9)$ & $91(46)$ \\
\hline
\end{tabular}
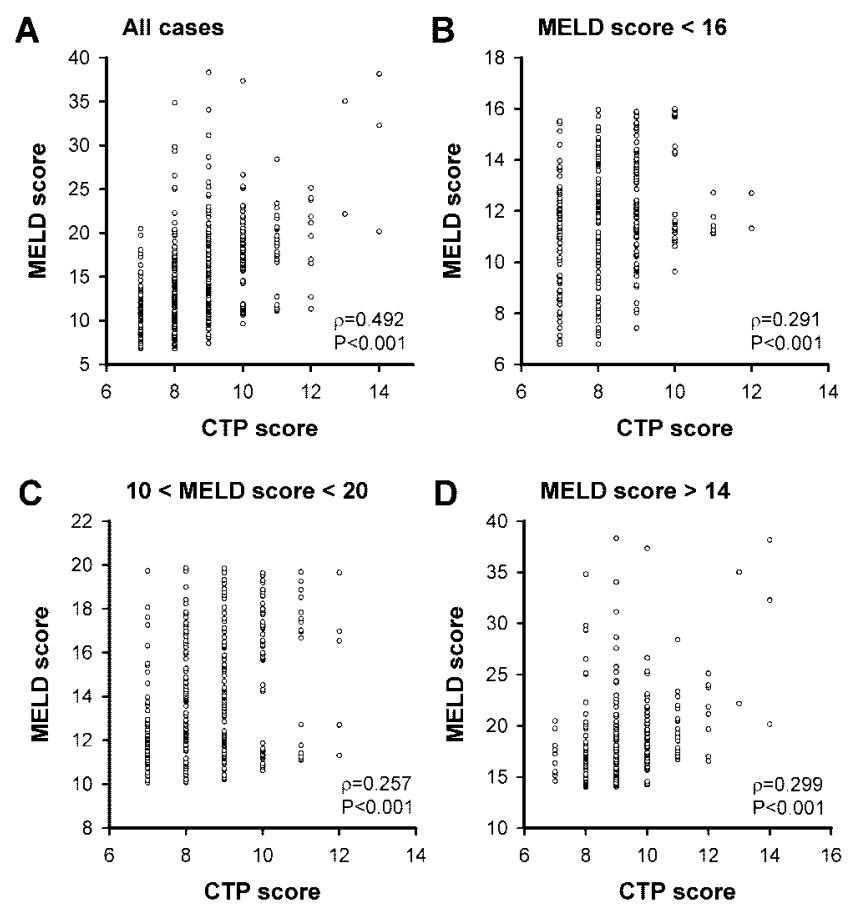

FIGURE 1. Correlation between MELD score and CTP score in all patients $(A)$, low $(<16)$ MELD (B), intermediate (10-20) MELD (C) and high (>14) MELD score group (D).

utilization of MELD system has been suggested to extend to patients with hepatocellular carcinoma (HCC), because the MELD score in combination with the staging system for HCC may further enhance the prognostic power and more accurately predict the survival for HCC patients undergoing arterial chemoembolization (18).

Consistent with most previous reports from our and other groups $(2-6,19)$, we found that MELD system has a significantly better ability than the CTP system to predict the short- and intermediate- term mortality by comparing the area under the ROC curve. Interestingly, by using the strategy that different distributions of MELD score blocks were tested in this study, we noted the ability of MELD for outcome prediction may not be adequately maintained. The predictive accuracy of MELD in patients with low or intermediate MELD scores was not better and sometimes could be worse than the CTP scoring system (Fig. 3). This result indicates that the MELD system is useful only in patients with relatively higher range MELD scores. Among those with lower MELD scores, the prognostic accuracy of the MELD system could be significantly impaired. A possible explanation is that patients with coexisting cirrhosis-related complications, such as ascites or encephalopathy, may frequently have a lower MELD score which does not necessarily reflect disease severity and poor prognosis $(20,21)$. As a result, additional prognostic markers, such as low serum sodium and ascites $(9,22,23)$, have been recently suggested to incorporate into the prognostic model to enhance the predictive accuracy of the MELD system especially in patients with low MELD scores. Taken together, our results show that the current MELD system has a potential limitation for outcome prediction because its predictive ability may not be uniform or homogeneous and is 
A
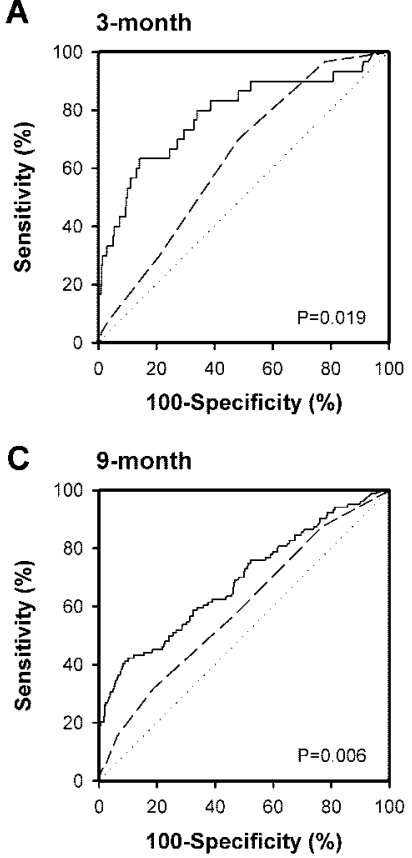

- MELD score
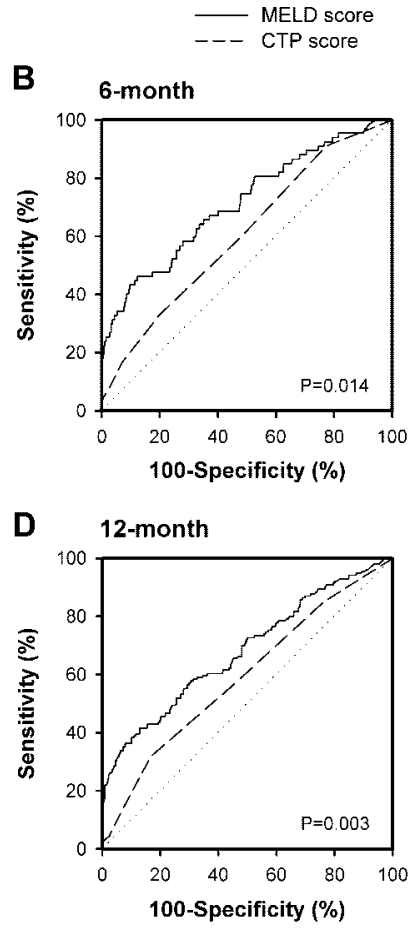

FIGURE 2. Comparison of the area under ROC curve between MELD score and CTP score in all patients for prediction of the risk of mortality at 3 months (A), 6 months (B), 9 months (C), and 12 months (D). MELD had a better predictive accuracy than the CTP system at all time periods.

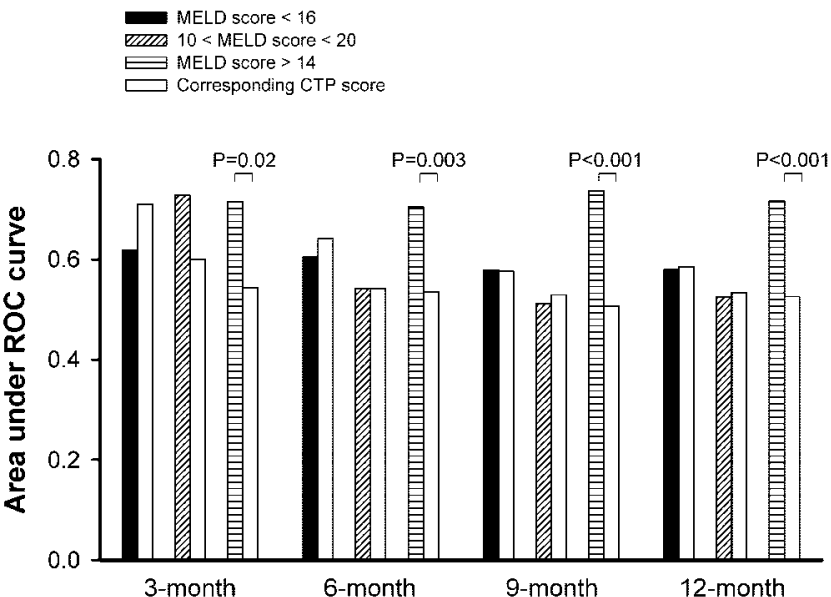

FIGURE 3. Comparison of the area under ROC curve between the MELD and CTP system for the prediction of mortality risk according to the distribution of MELD scores at different time frames. MELD was better than the CTP system only in patients with high (>14) MELD scores.

highly dependent on the distribution of the MELD scores in a given patient population.

Although it has been generally agreed that MELD is a useful model for organ allocation in liver transplantation, a previous study did not fully support its prognostic value because the CTP system was equally efficient in outcome prediction compared to the MELD system (10). It should be

mentioned that the mean MELD score of the patient population in that study was only 7 , and $32 \%$ of patients belonged to CTP class A liver reserve, suggesting a substantially high proportion of patients had very early stage cirrhosis (10). Nevertheless, it is not surprising that a score distribution composed of predominantly lower MELD scores may compromise its predictive ability. In fact, the prognostic function of the MELD system is characterized as a reverse $S$ wave by a sophisticated mathematical model indicated in a previous study (2), suggesting MELD works only partially in a population with mid- to lower range MELD scores. The risk of mortality at 3 months was quite low when the MELD score is below 20 and increases only slowly as the score increases. However, there is a rapid surge of the risk between the score range of 20 to 40 , and the mortality risk reaches a plateau when the MELD score is higher than 40 (2). Consistently, a recent report has shown that the survival benefit for patients undergoing liver transplantation pertains only to those who had MELD score of 18 or higher (24), indicating there is no demonstrable advantage for patients with a lower pretransplant MELD to receive a liver transplant possibly owing to an impaired predictive ability among these patients. Our results further provide a supporting mechanism and disclose an intrinsic drawback of the MELD system.

In this study, we have categorized three groups of patients with partially overlapped MELD scores. This approach has an advantage to mimic different clinical scenarios of the MELD scores that are distributed to the very right or left, or middle part of the distribution curve at a population level. It should be emphasized that each subgroup was treated independently and assessed individually to compare the predictive ability between MELD and CTP score (Fig. 3), and there has been no direct comparison for the clinical parameters among the three subgroups. By exploring the predictive value for different score distributions, our study provides important information that for a given study population containing predominantly a lower or higher range of MELD scores, the results of analysis might be entirely different.

The majority (73\%) of patients in this study had hepatitis B-related cirrhosis. This is quite different from the Western countries where alcoholism and hepatitis $C$ are more prevalent. A major concern is that the speed of disease progression might be faster due to persistent viral activity. Since all comparisons were made on the same background for the three MELD groups, the impact of different etiologies of liver cirrhosis on the interpretation of our results is considered minimal.

Another potential defect of MELD is that de novo development of HCC in cirrhotic patients awaiting liver transplantation may occur (25-27). The reported incidence was up to $20 \%$ of the cases (28), and an optimal MELD score has been proposed to be equivalent to the dropout rate on the waiting list (29). The occurrence of HCC can be a significant confounding factor to impair the prognostic power of MELD.

Although patients with a higher pretransplant MELD score had the greatest survival benefit (24), a high MELD score in itself has been reported to predict a poor survival in patients with hepatitis $\mathrm{C}$ at 2 years of liver transplantation (30). However, this finding could not be confirmed in our study because only a minority of patients had undergone liver transplantation during the study period. 
Our results confirm that MELD system has an overall better prognostic accuracy than the CTP scoring system. However, the outcome in patients with a lower range MELD scores cannot be reliably predicted solely with their MELD scores, and other alternative prognostic markers should be used in conjunction to compensate for this deficiency and enhance its predictive accuracy.

\section{REFERENCES}

1. Annual Report of the US Scientific Registry for Organ Transplantation and the Organ Procurement and Transplantation Network. Transplant Data 1990-1999. UNOS, Richmond, VA, and the Division of Transplantation, Bureau of Health Resources and Services Administration, US Department of Health and Human Services, Rockville, MD; 2000.

2. Wiesner R, Edwards E, Freeman R, et al. Model for end-stage liver disease (MELD) and allocation of donor livers. Gastroenterology 2003; 124: 91.

3. Freeman RB Jr., Wiesner RH, Roberts JP, et al. Improving liver allocation: MELD and PELD. Am J Transplant 2004; 4 Suppl 9: 114.

4. Olthoff KM, Brown RS Jr., Delmonico FL, et al. Summary report of a national conference: evolving concepts in liver allocation in the MELD and PELD era. Liver Transpl 2004; 10: A6.

5. Botta F, Giannini E, Romagnoli P, et al. MELD scoring system is useful for predicting prognosis in patients with liver cirrhosis and is correlated with residual liver function: a European study. Gut 2003; 52: 134.

6. Salerno F, Merli M, Cazzaniga M, et al. MELD score is better than Child-Pugh score in predicting 3-month survival of patients undergoing transjugular intrahepatic portosystemic shunt. J Hepatol 2002; 36: 494 .

7. Freeman RB. MELD: the holy grail of organ allocation? J Hepatol 2005; 42: 16 .

8. Mandell MS. Hepatopulmonary syndrome and portopulmonary hypertension in the model for end-stage liver disease (MELD) era. Liver Transpl 2004; 10 (10 Suppl 2): S54.

9. Heuman DM, Abou-Assi SG, Habib A, et al. Persistent ascites and low serum sodium identify patients with cirrhosis and low MELD scores who are at high risk for early death. Hepatology 2004; 40: 802.

10. Angermayr B, Cejna M, Karnel F, et al. Child-Pugh versus MELD score in predicting survival in patients undergoing transjugular intrahepatic portosystemic shunt. Gut 2003; 52: 879.

11. Papatheodoridis GV, Cholongitas E, Dimitriadou E, et al. MELD vs Child-Pugh and creatinine-modified Child-Pugh score for predicting survival in patients with decompensated cirrhosis. World J Gastroenterol 2005; 11: 3099.

12. Lucey MR, Brown KA, Everson GT, et al. Minimal criteria for placement of adults on the liver transplant waiting list: a report of a national conference organized by the American Society of Transplant Physicians and the American Association for the Study of Liver Diseases. Transplantation 1998; 66: 956.

13. Hanley JA, McNeil BJ. The meaning and use of the area under a receiver operating characteristic (ROC) curve. Radiology 1982; 143: 29.

14. Hanley JA, McNeil BJ. A method of comparing the areas under receiver operating characteristic curves derived from the same cases. Radiology 1983; 148: 839.

15. Wang VS, Saab S. Liver transplantation in the era of model for endstage liver disease. Liver Int 2004; 24: 1.

16. Freeman RB, Wiesner RH, Edwards E, et al. United Network for Organ Sharing Organ Procurement and Transplantation Network Liver and Transplantation Committee. Results of the first year of the new liver allocation plan. Liver Transpl 2004; 10: 7.

17. Said A, Williams J, Holden J, et al. Model for end stage liver disease score predicts mortality across a broad spectrum of liver disease. J Hepatol 2004; 40: 897.

18. Testa R, Testa E, Giannini E, et al. Trans-catheter arterial chemoembolisation for hepatocellular carcinoma in patients with viral cirrhosis: role of combined staging systems, Cancer Liver Italian Program (CLIP) and Model for End-stage Liver Disease (MELD), in predicting outcome after treatment. Aliment Pharmacol Ther 2003; 17: 1563.

19. Huo TI, Wu JC, Lin HC, et al. Evaluation of the increase in model for end-stage liver disease ( $\triangle$ MELD) score over time as a prognostic predictor in patients with advanced liver cirrhosis: risk factor analysis and comparison with initial MELD and Child-Turcotte-Pugh score. J Hepatol 2005; 42: 826.

20. Yoo HY, Edwin D, Thuluvath PJ. Relationship of the model for endstage liver disease (MELD) scale to hepatic encephalopathy, as defined by electroencephalography and neuropsychometric testing, and ascites. Am J Gastroenterol 2003; 98: 1395.

21. Huo TI, Wu JC, Lee SD. MELD in liver transplantation: the da Vinci code for the Holy Grail? J Hepatol 2005; 42: 474.

22. Biggins SW, Rodriguez HJ, Bacchetti P, et al. Serum sodium predicts mortality in patients listed for liver transplantation. Hepatology 2004; 41: 32 .

23. Ruf AE, Kremers WK, Chavez LL, et al. Addition of serum sodium into the MELD score predicts waiting list mortality better than MELD alone. Liver Transpl 2005; 11: 336.

24. Merion RM, Schaubel DE, Dykstra DM, et al. The survival benefit of liver transplantation. Am J Transplant 2005; 5: 307.

25. Llovet JM, Burroughs A, Bruix J. Hepatocellular carcinoma. Lancet 2003; 362: 1907.

26. Maddala YK, Stadheim L, Andrews JC, et al. Drop-out rates of patients with hepatocellular cancer listed for liver transplantation: outcome with chemoembolization. Liver Transpl 2004; 10: 449.

27. Ikeda K, Saitoh S, Koida I, et al. A multivariate analysis of risk factors for hepatocellular carcinogenesis: a prospective observation of $795 \mathrm{pa}-$ tients with viral and alcoholic cirrhosis. Hepatology 1993; 18: 47.

28. Van Thiel DH, Yong S, Li SD, et al. The development of de novo hepatocellular carcinoma in patients on a liver transplant list: frequency, size, and assessment of current screening methods. Liver Transpl 2004; 10: 631 .

29. Huo TI, Wu JC, Lin HC, et al. Determination of the optimal model for end-stage liver disease (MELD) score in patients with small hepatocellular carcinoma undergoing loco-regional therapy. Liver Transpl 2004; 10: 1507.

30. Onaca NN, Levy MF, Netto GJ, et al. Pretransplant MELD score as a predictor of outcome after liver transplantation for chronic hepatitis C. Am J Transplant 2003; 3: 626. 